\title{
Author Correction: Electronic correlations in twisted bilayer graphene near the magic angle
}

Youngjoon Choi, Jeannette Kemmer, Yang Peng, Alex Thomson, Harpreet Arora, Robert Polski, Yiran Zhang (D,

Hechen Ren, Jason Alicea, Gil Refael, Felix von Oppen, Kenji Watanabe (D), Takashi Taniguchi and Stevan Nadj-Perge ${ }^{D}$

Correction to: Nature Physics https://doi.org/10.1038/s41567-019-0606-5, published online 5 August 2019.

In the version of this Article originally published online, the funding support for J.A. from the Army Research Office was inadvertently left out of the Acknowledgements; the information has now been added to the penultimate sentence, which now ends "J.A. support from the Army Research Office under grant award W911NF-17-1-0323 and the NSF through grant DMR-1723367." All versions of this Article have been amended.

Published online: 25 October 2019

https://doi.org/10.1038/s41567-019-0724-0

๑ The Author(s), under exclusive licence to Springer Nature Limited 2019 